\title{
Endogenous natural and radiation-induced DNA lesions: differences and similarities and possible implications for human health and radiological protection
}

\author{
J.-L. Ravanat ${ }^{*}$ \\ Univ. Grenoble Alpes, CEA, CNRS, INAC, SYMMES, CIBEST, CEA Grenoble, 17 rue des Martyrs, 38054 Grenoble Cedex 9, France.
}

Received: 31 October 2018 / Accepted: 8 November 2018

\begin{abstract}
During the last few decades, a considerable amount of work has been done to better assess the effects of ionizing radiation on living organisms. In particular a lot of attention has been focused on the consequences of modifications of the DNA macromolecule, the support of the genetic information. Detailed information is now available on the formation of radiation-induced DNA lesions at the physical, chemical and biological levels. Emphasis will be placed in this review article on the differences and similarities, in term of DNA lesions formation and outcome, between endogenous oxidative stress and ionizing radiation, both stresses that could produce oxidative DNA lesions through similar mechanistic pathways involving mostly reactive oxygen species. If the chemical nature of the generated lesions is similar, the differences in term of biological consequences could be attributed to their spatial distribution in genomic DNA, since ionizing radiations produce lesions in cluster. These clusters of lesions represent a challenge for the DNA repair machinery. In contrast, endogenous oxidative stress generates scattered lesions that could be repaired with a much higher efficacy and fidelity. Possible implication of the use of DNA damage and repair for human health purposes and radiological protection will be discussed.
\end{abstract}

Keywords: DNA / radiation damage / biological indicator

\section{Introduction}

The DNA molecule that is the support of the genetic information could be damaged by both endogenous and exogenous stress (Lindahl, 1993). Since only one copy of this very large biomolecule is present in the nucleus of eukaryotic cells, in order to maintain the correct genetic information the DNA molecule has to be repaired when damaged, and its chemical structure has to be restored (Myles and Sancar, 1989). Indeed, our cells have to deal each day with that equilibrium between formation and repair of DNA lesions produced even in the absence of exogenous stress by at least endogenous oxidative stress (Marnett et al., 2003). Most of the time, the DNA repair machinery is able to repair with a high fidelity the generated DNA lesions. However, under certain conditions, for example following an overwhelming production of damage, or a reduced efficacy of repair, an accumulation of damages may occur and this may have severe biological consequences, including: mutations, chromosome instability, aging, cancer...

\footnotetext{
*Corresponding author: jravanat@cea.fr
}

Reactive oxygen species (ROS) are among the different stresses that could damage DNA, one of the most important. ROS are produced endogenously since they play a role in different enzymatic reactions and in the respiratory chain, but they could also be produced as side products. In addition, both non ionizing radiations including UV and visible light through photosensitization reactions, and ionizing radiation (IR) generate ROS. These ROS could damage all cellular constituents and among them the nucleic acids (Ravanat and Douki, 2016). A considerable amount of work has been done during the last three decades to study the reactions of ROS with the DNA macromolecule. Since the effects of IR could be mostly attributed to the initial production of ROS (socalled the indirect effect of radiation) subsequently to water radiolysis, the generated DNA damages are not chemically different in nature from those produced by endogenous oxidative stress.

The purpose of this paper is to make a review of the different effects of endogenous natural stress and of IR on the DNA molecule: type of lesions and repair mechanisms, mechanistic aspects of the lesions and measurement of DNA damage. According to the editorial line of Radioprotection (Bourguignon, 2017), this knowledge at the chemical forefront 


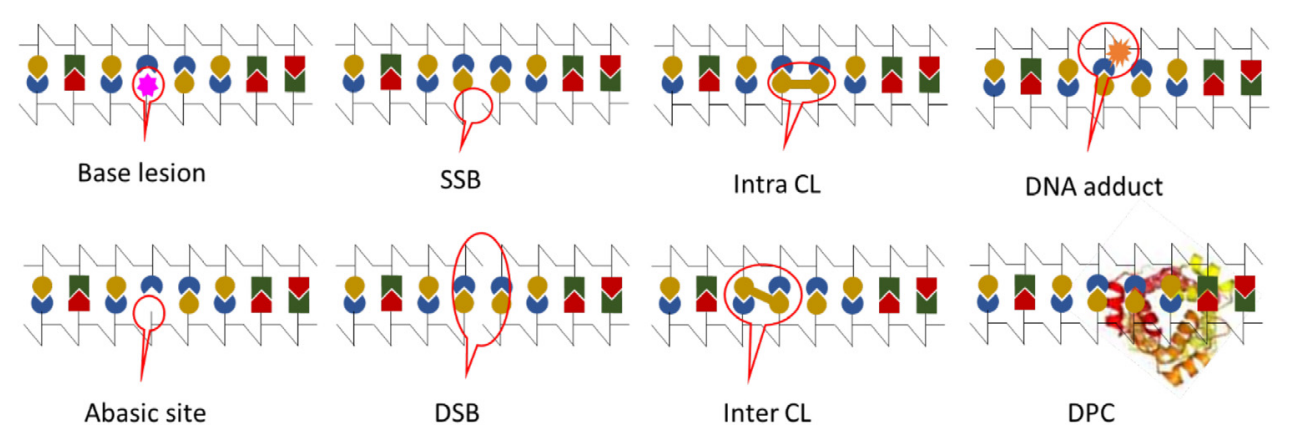

Fig. 1. Schematic representation of the different types of DNA lesions, including bases lesions, single strand breaks (SSB), double strand breaks (DSB), abasic sites, intra- and inter-strand cross links (CL), DNA adducts and DNA protein crosslinks (DPC).

of radiation biology is necessary for a proper interpretation of the subsequent health effects before drawing some perspectives in radiological protection.

\section{Different types of DNA lesions and repair mechanisms}

The lesions that are produced in genomic DNA following either an endogenous natural or exogenous stress could be classified into different categories (Fig. 1). A base lesion is defined when only the chemical structure of one of the four DNA bases is modified. A single strand break (SSB) corresponds to the break (of the sugar moiety or phosphodiester bond) of one of the two strands, whereas when the two strands are broken (separated by not more than two helix turns) a double strand break (DSB) is produced. Under certain conditions, the N-glycosidic bond between the DNA base and the 2-deoxyribose could be also broken, generating a so-called abasic site. Chemical bonds could also be produced between two DNA bases. If the two bases are located on the same strand, an intra-strand crosslink (CL) is produced, whereas if they are located on the opposite strand, the lesion corresponds to an inter-strand crosslinks. A chemical crosslink could also occur with another endo- or exogenous molecule to produce a DNA adduct, or also with surrounding proteins to produced DNA protein-crosslinks (DPC).

In cells, the different DNA lesions are not repaired with the same kinetic, and different repair systems exist to remove the modification and restore the native DNA structure (Myles and Sancar, 1989). When only one strand is modified, for example in the case of the formation of a base lesion (Fig. 2), the base excision repair (BER) system excises the modified base through hydrolysis of the $\mathrm{N}$-glycosidic bond (between deoxyribose and the base) to produce an abasic site (Schermerhorn and Delaney, 2014). The residual sugar residue is then removed by an endonuclease (AP Endo) leaving a gap in the DNA fragment. Using the information on the opposite strand (thanks to the complementary between DNA bases A with $\mathrm{T}$ and $\mathrm{G}$ with $\mathrm{C}$ ), a polymerase can then re-incorporate the natural missing base using the corresponding tri-phosphate derivative. For example, if guanine is oxidized into 8oxoguanine, the hOOG (human OxoGuanine Glycosylase) glycosylase removes the unnatural lesion, then creating an abasic site in front of a cytosine base (on the opposite strand). The polymerase (Pol) that recognizes the cytosine is then able

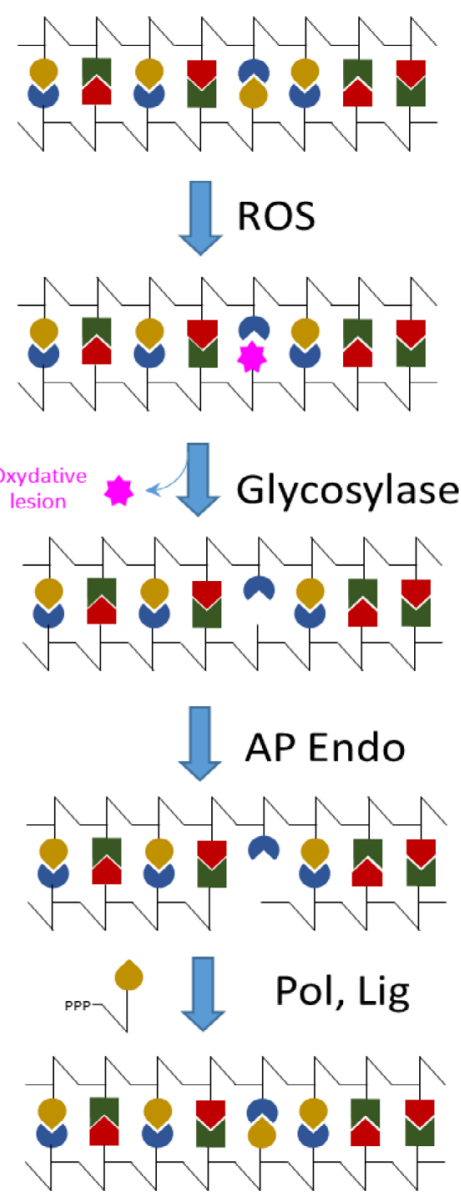

Fig. 2. Representation of the base excision repair (BER) mechanism of an oxidative DNA lesion.

to incorporate a guanine using dGTP, and finally the ligase (Lig) restores the DNA integrity.

The nature of the base on the complementary strand is thus a warranty for an accurate repair. However, depending on the nature of the lesions, presented in Figure 1, the information on the complementary strand could be also damaged, in particular when several lesions are produced in a close vicinity (so-called cluster lesions). Thus, this represents a challenge for the repair machinery.

BER repairs single strand breaks (SSB) and base lesions, nucleotide excision repair (NER) takes in charge intra-strand 


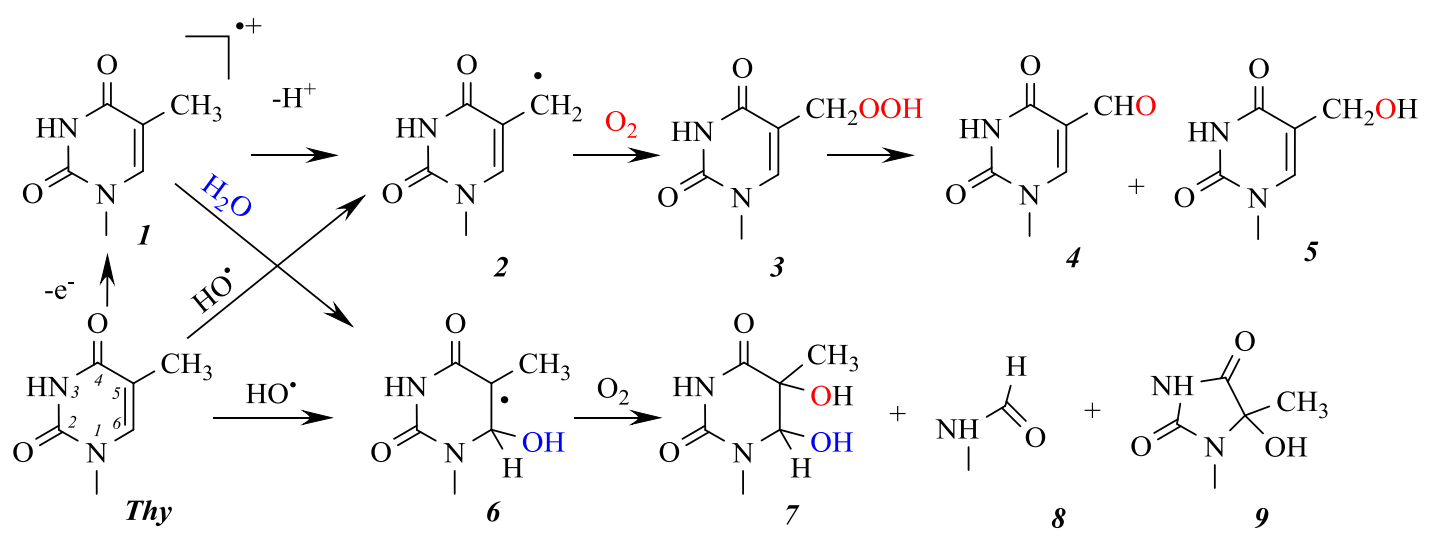

Fig. 3. Mechanism of radiation-induced decomposition of thymine (Thy) DNA base by both the direct and indirect effects of ionizing radiation.

cross-links (CL) (such as pyrimidine dimers produced by UV light), removing a short oligonucleotide containing the lesion (about 25 oligonucleotides in eukaryotes) and again using the opposite strand for an accurate repair.

It becomes obvious that repair of cluster lesions containing several damage on the two strands, including double strand breaks (DSB), represents a challenge for the cell for an accurate repair in order to restore the DNA integrity and its original sequence (Kakarougkas and Jeggo, 2014). Homologous recombination (HR), through the use of the sister chromatin is able to repair DSB with a high fidelity, but, nonhomologous end joining (NHEJ) restores a double helix by simply ligating the two DNA broken ends with possible changes in the original sequence and in the genetic code, the price to pay for a cell having DSB and undergoing replication.

\section{Mechanistic aspects of DNA lesions}

The biological effects of ionizing radiation is mediated by two different mechanisms, named the direct and indirect effects (Nikjoo, 1990). When the radiation affects directly (through ionization) the target molecule, in our case DNA, this is called the direct effect. In the indirect effect, the radiation interacts with water molecules thus creating water radiolysis, and the produced reactive species could then damage the DNA molecule. The relative proportion of this two effects is still a mater a debate but as it will be illustrated below, the chemical nature of the DNA lesions produced by these two effects are similar (Ravanat and Douki, 2016).

The direct effect of radiation induces a direct ionization of DNA. As an example for thymine (Thy, Fig. 3) one of the four DNA bases, this induces the formation of a thymine radical cation (1). This unstable radical decomposes either through deprotonation or hydration. Deprotonation gives rise to the 5-methyluracil radical (2) that following reaction with molecular oxygen generates 5-formyluracil (4) and 5-hydroxymethyluracil (5) through the transient formation of unstable 5-hydroperoxymethyluracil (3). Hydration occurs mostly at $\mathrm{C} 6$ producing a $\mathrm{C} 5$ centered radical (6) that following reaction with $\mathrm{O}_{2}$ produces mostly thymine glycols (7), formylamine (8) and hydantoïns (9) derivatives. Interestingly, reactions of hydroxyl radicals, the main ROS produced through water radiolysis (indirect effect of ionizing radiation) with thymine produces similar decomposition products but in different yields (Ravanat and Douki, 2016). Again two competitive reactions with thymine could occur with $\mathrm{HO}^{\circ}$, either by hydrogen abstraction reaction or through addition onto the base moiety. Hydrogen abstraction occurs on the methyl group of thymine, producing the 5-methyluracil radical (2) that gives rise to 5-formyluracil (4) and 5-hydroxymethyluracil (5) as described above. $\mathrm{HO}^{\circ}$ addition onto $\mathrm{C} 5-\mathrm{C} 6$ double bond of Thy produces mostly the $\mathrm{C} 5$ centered radical (6) at the origin of thymine glycols (7), formylamine (8) and hydantoïns (9) derivatives (Cadet et al., 2010).

Such an example illustrates that similar decomposition products are obtained following either the indirect or direct effects of radiation. Indeed, the direct effect produces a radical cation that could either deprotonate or react with water (hydration reaction). The deprotonated radical cation could be also produced by $\mathrm{H}$-abstraction mediated by $\mathrm{HO}^{\circ}$ produced by the indirect effect and the hydrated radical is produced by $\mathrm{HO}^{\circ}$ addition onto C5-C6 double bond. Thus, this explains why similar products are obtained by the two mechanisms. Such similarity has been reported for the reactivity of the four different DNA bases.

In double stranded DNA the reactivity of initially produced radicals is significantly affected. If the direct effect could ionize directly the four different DNA bases with an almost similar efficacy, since guanine has the lowest ionization potential among the DNA constituents, guanine is able to "chemically repair" the produced radical cations located on the other DNA bases, by an electron transfer reaction (from guanine to produced guanine radical cation). Such an electron transfer chemically repairs pyrimidine (cytosine and thymine) and adenine radical cations and thus the direct effect produces almost exclusively guanine lesions such as 8-oxo-7,8-dihydro2'-deoxyguanosine (8-oxodGuo) the most studied DNA lesion. The specific formation of 8 -oxodGuo by the direct effect (confirmed experimentally using a high intensity UV laser irradiation that produces ionization of DNA by a twophoton absorption mechanism) has been used to determine the variation of the proportion of the direct and indirect effect according to the radiation quality (Douki et al., 2006). Indeed, an increase of the direct effect of radiations is expected with the increase of the linear energy transfer (LET) of radiations, and thus a higher proportion of 8-oxodGuo compared to other base lesions should occur. Experimentally such an increase 

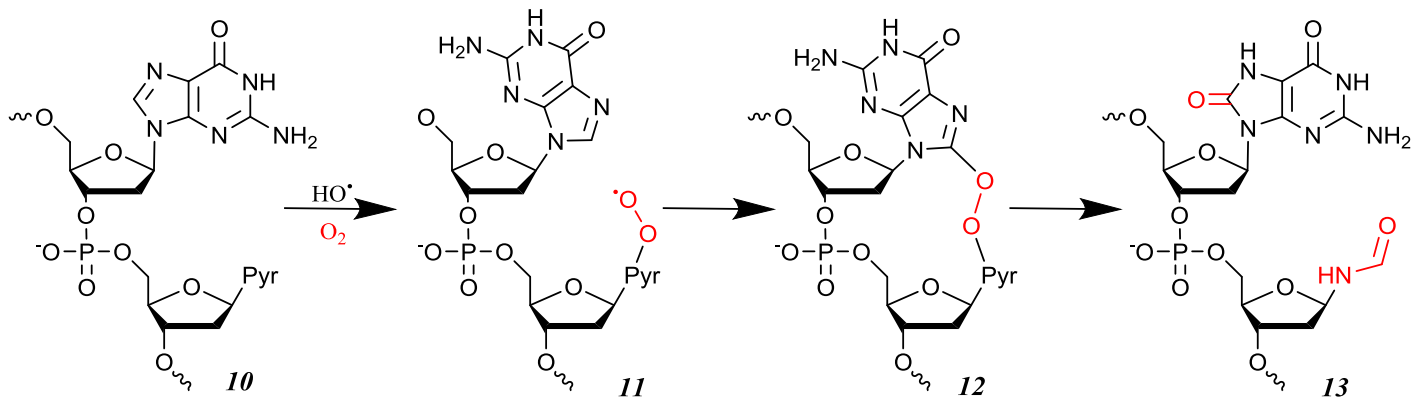

Fig. 4. Mechanism of formation of a tandem DNA lesions 8-oxodGuo-dF by a single oxidation event involving a peroxyl radical (11).

Clean DSB

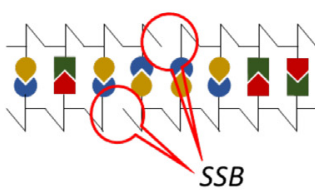

Pro DSB

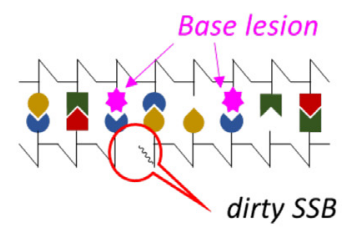

Dirty DSB

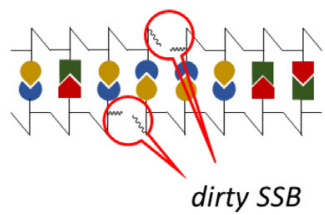

Complex DSB

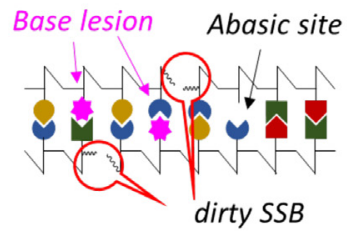

$\underline{\text { Tandem lesion }}$

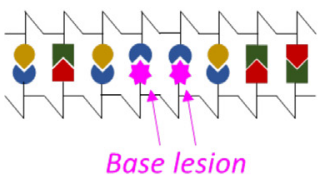

Non-DSB cluster lesion

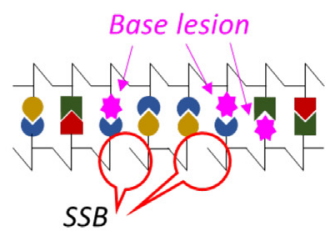

Fig. 5. Examples of different types of complex DNA lesions, including clean and dirty DSB, tandem lesion, pro-DSB, complex DSB and nonDSB cluster lesion.

was not observed, strongly suggesting that the indirect effect of radiation is predominant even for high LET radiation. This is in contrast to what is generally admitted i.e. the proportion of the direct effect is about $30 \%$ for low LET radiations and is increased when LET increases.

Another important aspect of the reactivity of DNA radicals that has to be considered in double stranded DNA is the possibility for the initially produced radical to react with surrounding DNA constituents. This was first reported using short oligonucleotides (small pieces of single strand DNA fragments) in which formation of double lesions (or so-called tandem lesions constituted of two adjacent DNA lesions) has been reported (Box et al., 1998). It was demonstrated that these tandem lesions could be produced at purine-pyrimidine sequences (10, Fig. 5) by a single ionization event. Their formation is explained by reaction of the initially generated peroxyl radical (11) with an adjacent DNA base to produce an unstable endoperoxide (12) that following decomposition gives rise to a tandem lesion containing 8-oxodGuo and formylamine (dF) (Bourdat et al., 2000) and additional tandem lesions (Bergeron et al., 2010). It was shown that these lesions are produced linearly with the irradiation dose (as expected due to the involvement of only one ionization event), and a strong sequence effect was observed. Indeed, the predominant formation of the tandem lesion with 8-oxodGuo located at the $5^{\prime}$ position of the modified pyrimidine (including $\mathrm{dF}$ ) is explained by the predominant addition of the pyrimidine radical $\left(\right.$ Pyr $^{\circ}$, Fig. 4) with a purine base located on its relative 5 ' position (Dupont et al., 2013). Interestingly, in double stranded DNA only $10 \%$ of the produced 8 -oxodGuo is generated by direct addition of $\mathrm{HO}^{\circ}$ onto guanine base, the other $90 \%$ are produced by DNA radicals that could either induce a one-electron oxidation reaction (mimicking the direct effect) or by addition of a peroxyl onto guanine (Cadet et al., 2012b; Ravanat et al., 2014). In addition, these complex lesions are more difficult to repair compared to single lesions, and for example, 8-oxodGuo involved in tandem lesions is less efficiently excised by its DNA repair glycosylase (Fpg or hOGG1) than a single 8-oxodGuo surrounding by only normal bases (Bergeron et al., 2010).

\section{DNA damage measurement}

Several complementary approaches have been developed for measuring and quantifying radiation-induced DNA lesions in cells (Cadet et al., 2011). The different methods measure either direct modification of the DNA (including strand breaks, modified DNA bases...) or indirectly DNA modifications through the activation of the DNA repair machinery, so called the DNA Damage Response (DDR). These two approaches, which will be described in more details below have their own advantages and limitations, including specificity, sensitivity, and the quantitative aspects of the measurement. In addition, 
effects of radiations on the DNA molecule could be estimated by measuring genomic instability (Mothersill and Seymour, 1998).

Physicochemical methods could be used to measure DNA modifications subsequently to DNA extraction from the irradiated cells. Gel electrophoresis that could separate DNA fragments according to their size could thus measure DNA strand breaks but this method is not very sensitive for measuring strand breaks in genomic DNA. Pulse field gel electrophoresis has been developed for the specific detection of DSB but not biologically relevant high doses of irradiation are required to measure these lesions in irradiated cells. Analytical chemistry has been also applied in the field of radiation biology (Ravanat, 2012). For such an approach, the DNA macromolecule extracted from cells has to be converted into its monomeric units, either DNA bases or nucleosides. Then, the different monomeric units are separated by a chromatographic method coupled to a sensitive detector that could specifically detect one or several DNA modifications. High performance liquid chromatography coupled to an ElectroChemical detector (HPLC-EC) has been extensively used for the detection of 8-oxodGuo (following enzymatic digestion of DNA into nucleosides), taking advantage of the low oxidation potential of that DNA lesion. Gas-chromatography coupled to mass spectrometry (GC-MS) subsequently to acid hydrolysis of DNA, was also developed but was shown to overestimate the levels of the oxidative DNA lesions due to artifactual oxidation of normal base occurring during the work-up (Cadet et al., 2012a). However, mass spectrometry is more versatile than the electrochemical detection that is limited to a few specific DNA lesions. Significant progresses have been made recently when HPLC was coupled to tandem mass spectrometry (HPLC-M/MS) thanks to the development of electrospray ionization. Nowadays, HPLC-MS/MS allows to detect and quantify less than one DNA modification per million normal DNA bases using a few $\mu \mathrm{g}$ of DNA (Douki et al., 2003). However, the limitation of that approach that requires DNA extraction and digestion, it that DNA oxidation may occur during the work-up procedure and thus measured levels of oxidative DNA lesions could be overestimated, as reported for the GC-MS assay. However, nowadays, optimized protocols have been developed to minimize such a spurious DNA oxidation to occur (Ravanat et al., 2002), and the method present the advantage of measuring and quantifying several well-defined DNA lesions simultaneously, and could be also used to search for new radiation-induced DNA lesions (Regulus et al., 2004). On the other hand, that approach requiring hydrolysis of the DNA polymer could not provide any information on the relative localization of the different lesions, thus making the detection of cluster lesions impossible. In addition, sensitivity for detection of radiation-induced DNA lesions is limited by the presence of endogenous oxidative damage that are chemically similar to those produced by ionizing radiation. As an example, in eukaryotic cells, a dose of about $40 \mathrm{~Gy}$ is required to double the endogenous level of 8-oxodGuo.

The comet assay or single cell gel electrophoresis method has been also extensively used and is very sensitive for the detection of SSB using a very low amount of biological material (Collins et al., 2008). Practically, isolated cells embedded in an agarose gel are lysed by a chemical treatment under alkaline conditions and the obtained isolated nuclei are exposed to an electric field. Broken DNA fragments migrate in the gel and then the fluorescently labeled DNA looks like a "comet", the proportion of DNA in its tails being proportional to the number of breaks. The method has been upgraded with the use of DNA glycosylases to allow the measurement of oxidative DNA bases, in addition to SSB and alkali-labile lesions, the DNA repair enzymes being able to convert an oxidized base into an additional strand break (Fig. 2). The assay is sensitive enough to detect lesions for doses below 0.1 Gy (Pouget et al., 2002). In addition, that method could be used under neutral conditions to measure only DSB, or can be adapted for the measurement of DNA repair capacities of cells extracts (Azqueta et al., 2014). The limitation is that the assay is not quantitative and that DNA glycosylases having a broad substrate specificity, the chemical nature of the measured lesions is not precisely determined. Also, cluster of lesions could not be detected using the comet assay.

During the last three decades, attention has been paid to the induction of the DDR and its possible use for DNA damage detection. Indeed, following irradiation, cells activate the DNA damage response and in particular kinases (ATM, ATR) that initiates the DDR. For example, ATM phosphorylates histone $\mathrm{H} 2 \mathrm{Ax}$ (a variant of histone $\mathrm{H} 2 \mathrm{~A}$ ) around the site of a DSB and this represents the first step for the recruitment of DNA repair enzymes. The use of specific antibodies raised against the phosphorylated form of $\mathrm{H} 2 \mathrm{Ax}$ variant, named $\gamma$-H2Ax allows to directly determine the number of foci in cells that are considered to represent the number of strand breaks (Lobrich et al., 2010). Foci of other DNA repair enzymes or proteins involved in DDR could be also used to detect damage for very low doses of radiation. The still remaining question is the specificity of that detection and if one $\gamma$-H2Ax foci represents only one DSB. Moreover, such similar approach but using fluorescently labeled DNA repair enzymes, allows to follow just after irradiation the kinetic of recruitment (and release) or DNA repair proteins at the sites of the damage DNA. This has been successfully applied for example to determine the nature of the DNA repair enzymes and their order of recruitment and release at DNA damage sites produced by high LET particles (Jakob and Taucher-Scholz, 2017). In addition, co-localization of $\gamma-\mathrm{H} 2 \mathrm{Ax}$ and OGG foci could be used to detect cluster lesions having both a DSB and an 8-oxodGuo.

\section{Double strand breaks (DSB) and cluster lesions}

During the last three decades a lot of work has been focused on the formation of double strand breaks (DSB) and in more general so-called clusters of lesions (Sage and Shikazono, 2017). Indeed, such lesions are considered the most important ones in terms of biological effects since they are a challenge for the DNA repair machinery. In addition, the interest in these kinds of DNA damage has been indirectly favored by the availability of a very sensitive assay to monitor them in cells $(\gamma \mathrm{H} 2 \mathrm{Ax}$ Foci). These clusters of lesions or "multiply damages site" are specifically produced by ionizing radiation and only poorly by endogenous oxidative stress (Lomax et al., 2002; Eccles et al., 2011). Indeed, to generate a DSB, two single strand breaks (SSB) on the two opposite 
strands have to be produced in a close vicinity (one or two helix turns, thus about 15-20 DNA base pairs apart). It could be considered that endogenous oxidative stress produces ROS randomly and thus the probability to produce two oxidation reactions in a close vicinity is very low. For ionizing radiations, ROS are produced along the particles track, and the density of ionization increases when the LET increases, thus explaining why DSB are produced by ionizing radiations and also why their proportion relatively to SSB increases with LET. However, the yield of radiation induced DSB formation is relatively low compared to isolated lesions including SSB and oxidative DNA lesions (Nikitaki et al., 2015). As mentioned above these DSB are detected using $\gamma$-H2Ax foci and it has been shown that their formation is linear with the irradiation dose, even for doses as low as $1 \mathrm{mGy}$ (Lobrich et al., 2010). However, the question of the specificity of the detection of DSB is still a matter of debate. Does one focus representing the phosphorylation of Histone $\mathrm{H} 2 \mathrm{Ax}$ over mega base pairs correspond to exactly one DSB? Could a non-DSB cluster lesion (for example a base damage and a SSB on the opposite strand) also induce the DDR and produce a $\gamma$-H2Ax focus? In addition to that, there is a complexity of cluster lesions (Fig. 5) and even of DSB, and thus their property to induce the DDR, and their efficacy of repair, might be different. One could imagine that so-called "clean DSB" (Fig. 5), produced by hydrolysis of the phosphodiester bonds, as those obtained using restriction enzymes, are much easier to repair than so-called "dirty DSB" that contained residual modified sugar residues produced by reaction of the 2-deoxyribose with hydroxyl radicals. This is even more complex since it has been also reported that DSB could be produced through thermolabile DNA lesions that are generated during irradiation as non DSB lesions but that could decompose later on to give rise to DSB (Cheng et al., 2015). Moreover, the repair by a DNA glycosylase of a cluster lesion constituted by (at least) a single strand break opposite to an oxidative DNA lesion could also give rise to a DSB delayed from the irradiation (Eccles et al., 2010). This is also the case when a SSB is present at a replication fork. This illustrates the complexity of the DNA lesions that could be produced during (or even delayed from) the irradiation. It would be surprising that all these different types of cluster lesions induce a similar DNA damage response and are repaired with the same kinetic, efficacy and fidelity...

\section{DNA lesions, human health and radiological protection}

This review of endogenous natural and radiation-induced DNA lesions shows that they are similar in their chemical nature. The classical difference made between direct and indirect lesions of IR does not hold so much anymore since the chemical pathways are similar at the DNA level. However, DSBs and cluster lesions are more likely due to IR as a result of the clustered deposition of energy than due to endogenous ROS.

In this review, we did not address the issue of the number of lesions produced by endogenous ROS and IR respectively since these numbers are highly variable and depend on the situations, i.e., physical stress, inflammation, infections, chronic diseases... On the one hand, exposure to IR from various origins and at different doses and dose rates on the other hand. Thus comparing these numbers is a completely different issue not in the scope of this paper.

The idea of using DNA lesions as biomarkers of a genotoxic agent is not very new, in particular for oxidative stress (Ravanat et al., 2012). Regarding the chemical effects of IR, since it has been shown that the formation of DNA lesions is linear with the irradiation dose, it could be proposed to use them as biomarkers of exposure (Hall et al., 2017). In fine the evaluation of DNA lesions whatever their origin is a way to assess the overall stress of cells.

However, there are numerous limitations.

First, if formation of DNA lesions is linear with the irradiation dose, lesions are not stable over time since cells try to repair them as soon as possible. So the number of lesions decreases after the end of the exposure and kinetic of repair depends on the nature of the lesions. For example, SSB are repaired within few minutes after irradiation, whereas complete repair of DSB requires at least $24 \mathrm{~h}$.

In addition, up to now there is no radiation-specific DNA lesions and as indicated above, lesions produced by radiations are chemically similar to those produced by endogenous oxidative stress or any compound that could generate an oxidative stress. Altogether, this illustrates the complexity of using DNA lesions as biomarkers of irradiation (Pouget et al., 2018). Furthermore, the yield of lesions produced by IR is very low in practice, and thus measuring these lesions in people exposed to low doses of radiation is very challenging. However, DNA DSBs have been evaluated as $\gamma \mathrm{H} 2 \mathrm{AX}$ foci in a low dose ex vivo experiment with human mammary epithelium mimicking mammography exposures in the range of few mGy (Colin et al., 2011). Besides spontaneous foci before any IR exposure, a dose effect, a repeated dose effect and a late induced effect were observed and found more prominent in women with high familial risk of breast cancer. Thus, DNA DSB at low irradiation dose do exist!

On another hand, when DNA lesions are not repaired correctly, they can induce cytogenetic alterations, especially dicentric chromosomes (DC) in the case of IR. Thus, measuring the number of DC could detect recent exposure but to a dose of about $0.1 \mathrm{~Gy}$ or more and this represents nowadays the gold reference for biodosimetry. Already in early eighties an increase in chromosomic aberrations have been measured in infants and children following angiocardiography (Adams et al., 1978), this was attributed to the increase dose deposition mediated by the contrast agent. Such an effect is now used for therapeutic purpose (Santos et al., 1983). However, the threshold of $0.1 \mathrm{~Gy}$ is rather high because of the natural presence of dicentric chromosomes. The main radiological protection issue today is related to the exposures to low dose IR, e.g., medical exposures. We have seen above that both DNA DSBs and dicentric chromosomes are naturally observed and both of them can be due to IR exposures and to other endogenous or exogenous genotoxic stress as well. Thus it becomes even harder to differentiate the responsibility of endogenous ROS and exogenous ROS in the creation of DNA lesions. The use of DNA lesions as biomarkers of exposure to low doses of radiation would require the identification of a specific modification of a biological constituent (potentially DNA but not only) that is not produced by other stress including endogenous of course oxidative stress. Does this 
specific biomarker exist? This is not sure. The other strategy could be to measure a global cellular response (genes induction for example) to different stress, including IR, hoping that each stress could induce a specific response (Nikitaki et al., 2015) representing a signature of the original stress. We might expect that the formation and/or induced cell response of the radiation-specific clustered lesions could be used in the future as specific biomarkers of radiation exposure even for very low doses, but additional works have to be done to achieve such a challenging goal.In this context another emerging and critical issue is the monitoring of the DNA repair efficacy to estimate the individual response to IR with its two features radiosensitivity and radiosusceptibility (Foray et al., 2016). Among the patients who are treated by radiotherapy, some of them demonstrate a high radiosensitivity as severe side effects or complications due to damage to healthy cells and tissues and not related to an error in dose delivery. This damage may be involved in the later induction of secondary cancers in radiosusceptible patients. On the opposite, some tumors are very radioresistant and thus the irradiation will not have the expected therapeutic effect. Thus it becomes evident that measuring the radio resistance or radiosensitivity of patients could allow to optimize their therapeutic treatment. At least a part of the radioresistance or radiosensitivity could be attributed to the efficacy of DNA repair, thus measuring DNA repair capacities could be a marker of radiosensitivity, e.g., pATM nucleus assay (Vogin et al., 2018). The DNA repair machinery, involving several repair systems, and numerous different (probably also including still unknown) enzymes, measuring differences at the gene level seems not convenient, except for rare extreme cases of mutation in important repair enzymes for example for Xeroderma Pigmentosum, Cokaine Syndrom or Ataxia Telangiectasia Mutated (ATM) patients (O'Driscoll, 2012). In addition, efficacy of repair depends not only on the repair machinery, but also on its induction through the DNA damage response (DDR). Several approaches have been developed to measure either DNA repair efficacy or induction of DDR. This could represent the future for personalized medicine but raises the ethical question of testing the individual response of workers potentially exposed to IR.

In conclusion, although we do know a lot on the chemistry of endogenous natural and radiation-induced DNA lesions, it is obvious that further research is necessary to better understand the mechanisms of their signalization and repair, and to be able to fully differentiate their effects on health. Finally one should also keep in mind that the harmful effects of IR on DNA are positively used for therapeutic purposes, for instance to treat cancer using radiotherapy alone or in efficient multi therapeutic approaches combining the use of radiation and DNA repair inhibitors.

\section{References}

Adams FH, Norman A, Bass D, Oku G. 1978. Chromosome damage in infants and children after cardiac catheterization and angiocardiography. Pediatrics 62: 312-316.

Azqueta A, Slyskova J, Langie SA, O’Neill Gaivao I, Collins A. 2014. Comet assay to measure DNA repair: approach and applications. Front. Genet. 5: 288.
Bergeron F, Auvré F, Radicella JP, Ravanat J-L. 2010. $\mathrm{HO}^{\circ}$ radicals induce an unexpected high proportion of tandem base lesions refractory to repair by DNA glycosylases. Proc. Natl. Acad. Sci. U.S.A. 107: 5528-5533.

Bourdat A-G, Douki T, Frelon S, Gasparutto D, Cadet J. 2000. Tandem base lesions are generated by hydroxyl radical within isolated DNA in aerated aqueous solution. J. Am. Chem. Soc. 122: 4549-4556.

Bourguignon M. 2017. Radioprotection, the way forward. Radioprotection 52: 227.

Box HC, Budzinski EE, Dawidzik JB, Wallace JC, Iijima H. 1998. Tandem lesions and other products in X-irradiated DNA oligomers. Radiat. Res. 149: 433-439.

Cadet J, Douki T, Ravanat J-L. 2010. Oxidatively generated base damage to cellular DNA. Free Radic. Biol. Med. 49: 9-21.

Cadet J, Douki T, Ravanat J-L. 2011. Measurement of oxidatively generated base damage in cellular DNA. Mutat. Res. 711: 3-12.

Cadet J, Douki T, Ravanat J-L, Wagner R. 2012a. Measurement of oxidatively generated base damage to nucleic acids in cells: facts and artifacts. Bioanalytical Rev. 4: 55-74.

Cadet J, Ravanat J-L, Taverna-Porro M, Menoni H, Angelov D. 2012b. Oxidatively generated complex DNA damage: tandem and clustered lesions. Cancer letters 327: 5-15.

Cheng Y, Li F, Mladenov E, Iliakis G. 2015. The yield of DNA double strand breaks determined after exclusion of those forming from heat-labile lesions predicts tumor cell radiosensitivity to killing. Radiother. Oncol. 116: 366-373.

Colin C, Devic C, Noel A, Rabilloud M, Zabot MT, Pinet-Isaac S, Giraud S, Riche B, Valette PJ, Rodriguez-Lafrasse C, Foray N. 2011. DNA double-strand breaks induced by mammographic screening procedures in human mammary epithelial cells. Int. J. Radiat. Biol. 87: 1103-1112.

Collins AR, Oscoz AA, Brunborg G, Gaivao I, Giovannelli L, Kruszewski M, Smith CC, Stetina R. 2008. The comet assay: topical issues. Mutagenesis 23: 143-151.

Douki T, Ravanat J-L, Frelon S, Bourdat A-G, Pouget J-P, Cadet J. 2003. HPLC-MS/MS measurement of oxidative base damage to isolated and cellular DNA. In: Critical reviews of oxidative stress and aging: Intervention (RG. Cutler, H. Rodriguez, Eds.), pp. 190-202. Singapore: Wold Scientific publisher.

Douki T, Ravanat J-L, Pouget J-P, Testard I, Cadet J. 2006. Minor contribution of direct ionization to DNA base damage induced by heavy ions. Int. J. Radiat. Biol. 82: 119-127.

Dupont C, Patel C, Ravanat JL, Dumont E. 2013. Addressing the competitive formation of tandem DNA lesions by a nucleobase peroxyl radical: a DFT-D screening. Org. Biomol. Chem. 14: 3038-3045.

Eccles LJ, Lomax ME, O’Neill P. 2010. Hierarchy of lesion processing governs the repair, double-strand break formation and mutability of three-lesion clustered DNA damage. Nucleic Acids Res. 38: 1123-1134.

Eccles LJ, O’Neill P, Lomax ME. 2011. Delayed repair of radiation induced clustered DNA damage: friend or foe? Mutat. Res. 711: 134-141.

Foray N, Bourguignon M, Hamada N. 2016. Individual response to ionizing radiation. Mutat. Res. 770: 369-386.

Hall J, Jeggo PA, West C, Gomolka M, Quintens R, Badie C, Laurent O, Aerts A, Anastasov N, Azimzadeh O, Azizova T, Baatout S, Baselet B, Benotmane MA, Blanchardon E, Guéguen Y, Haghdoost S, HarmsRinghdahl M, Hess J, Kreuzer M, Laurier D, Macaeva E, Manning G, Pernot E, Ravanat J-L, Sabatier L, Tack K, Tapio S, Zitzelsberger H, Cardis E. 2017. Ionizing radiation biomarkers in epidemiological studies - An update. Mutat. Res./Rev. Mutat. Res. 771: 59-84. 
Jakob B, Taucher-Scholz G. 2017. Live cell imaging to study realtime atm-mediated recruitment of DNA repair complexes to sites of ionizing radiation-induced DNA damage. Methods Mol. Biol. 1599: 287-302.

Kakarougkas A, Jeggo PA. 2014. DNA DSB repair pathway choice: an orchestrated handover mechanism. Br. J. Radiol. 87: 20130685.

Lindahl T. 1993. Instability and decay of the primary structure of DNA. Nature 362: 709-715.

Lobrich M, Shibata A, Beucher A, Fisher A, Ensminger M, Goodarzi AA, Barton O, Jeggo PA. 2010. GammaH2AX foci analysis for monitoring DNA double-strand break repair: strengths, limitations and optimization. Cell Cycle 9: 662-669.

Lomax ME, Gulston MK, O'Neill P. 2002. Chemical aspects of clustered DNA damage induction by ionising radiation. Radiat. Prot. Dosim. 99: 63-68.

Marnett LJ, Riggins JN, West JD. 2003. Endogenous generation of reactive oxidants and electrophiles and their reactions with DNA and protein. J. Clin. Invest. 111: 583-593.

Mothersill C, Seymour CB. 1998. Mechanisms and implications of genomic instability and other delayed effects of ionizing radiation exposure. Mutagenesis 13: 421-426.

Myles GM, Sancar A. 1989. DNA repair. Chem. Res. Toxicol. 2: $197-$ 226.

Nikitaki Z, Hellweg CE, Georgakilas AG, Ravanat J-L. 2015. Stressinduced DNA damage biomarkers: Applications and limitations. Frontiers Chem. 3: 35

Nikjoo H. 1990. Mechanism of DNA damage by direct and indirect effects $-\mathrm{a}$ review of experimental and theoretical data. Int. J. Radiat. Biol. 57: 1257-1257.

O'Driscoll M. 2012. Diseases associated with defective responses to DNA damage. Cold Spring Harb. Perspect. Biol. 4: a012773a012773.

Pouget J-P, Frelon S, Ravanat J-L, Testard I, Odin F, Cadet J. 2002. Formation of modified DNA bases in cells exposed either to gamma radiation or to high-LET particles. Radiat. Res. 157: 589-595.

Pouget JP, Georgakilas AG, Ravanat JL. 2018. Targeted and off-target (Bystander and Abscopal) Effects of radiation therapy: Redox mechanisms and risk/benefit analysis. Antioxid. Redox Signal. 29: 1447-1487.

Ravanat JL. 2012. Chromatographic methods for the analysis of oxidatively damaged DNA. Free Radic. Res. 46: 479-491.

Ravanat JL, Douki T. 2016. UV and ionizing radiations induced DNA damage, differences and similarities. J. Rad. Phys. Chem. 128: 92-102.

Ravanat JL, Douki T, Duez P, Gremaud E, Herbert K, Hofer T, Lasserre L, Saint-Pierre C, Favier A, Cadet J. 2002. Cellular background level of 8-oxo-7,8-dihydro-2'-deoxyguanosine: an isotope based method to evaluate artefactual oxidation of DNA during its extraction and subsequent work-up. Carcinogenesis 23: 1911-1918.

Ravanat JL, Cadet J, Douki T. 2012. Oxidatively generated DNA lesions as potential biomarkers of in vivo oxidative stress. Curr. Mol. Med. 12: 655-674.

Ravanat JL, Breton J, Douki T, Gasparutto D, Grand A, Rachidi W, Sauvaigo S. 2014. Radiation-mediated formation of complex damage to DNA: a chemical aspect overview. Br. J. Radiol. 87: 20130715

Regulus P, Spessotto S, Gateau M, Cadet J, Favier A, Ravanat J-L. 2004. Detection of new radiation-induced DNA lesions by liquid chromatography coupled to tandem mass spectrometry. Rapid. Commun. Mass. Spectrom. 18: 2223-2228.

Sage E, Shikazono N. 2017. Radiation-induced clustered DNA lesions: Repair and mutagenesis. Free Radic. Biol. Med. 107: 125-135.

Santos Mello R, Callisen H, Winter J, Kagan AR, Norman A. 1983. Radiation dose enhancement in tumors with iodine. Med. Phys. 10: $75-78$.

Schermerhorn KM, Delaney S. 2014. A chemical and kinetic perspective on base excision repair of DNA. Acc. Chem. Res. 47, $1238-1246$.

Vogin G, Bastogne T, Bodgi L, Gillet-Daubin J, Canet A, Pereira S, Foray N. 2018. The phosphorylated ATM immunofluorescence assay: A high-performance radiosensitivity assay to predict postradiation therapy overreactions. Int. J. Radiat. Oncol. Biol. Phys. 101: 690-693.

Cite this article as: Ravanat J-L. 2018. Endogenous natural and radiation-induced DNA lesions: differences and similarities and possible implications for human health and radiological protection. Radioprotection 53(4): 241-248 